\title{
Table ronde 6
}

\section{Quelles sont \\ les responsabilités \\ de la société vis-à-vis \\ des personnes atteintes \\ de maladies rares?}

Participent à la table ronde :

Florence Bordon-Pallier, Genzyme

Brigitte Chabrol, Neuro-pédiatre, Aix-Marseille Université

Pierre Le Coz, philosophe, espace éthique méditerranéen/UMR 7268 ADES/

AMU/EFS/CNRS

Anne-Sophie Lapointe, Eurordis/VML

Vololona Rabeharisoa, sociologue, PSL MINES ParisTech

aux dépens d'autres sujets. Les ressources n'étant pas illimitées, il importe de savoir qui décide d'allouer les ressources disponibles à telle ou telle maladie. En fait, nous ne savons pas.

Ce ne sont pas les patients, qui sont en concurrence avec d'autres malades. Ce ne sont pas les cliniciens, qui sont les professionnels de première ligne. Ce ne sont pas non plus les industriels, qui sont pourtant des partenaires essentiels de la santé publique. Ce ne sont pas les politiques ni le législateur, qui suivent théoriquement ce que souhaite la population. En fait, c'est la Société qui décide. Comme la décision appartient à tout le monde, le risque serait de penser qu'elle n'appartient à personne. Ce n'est pas vrai. La Société est un ensemble de groupes de personnes qui s'expriment de façon extrêmement diffuse et complexe. Toute personne a le droit d'exprimer son point de vue et de le porter devant le décideur - cela s'appelle le lobbying, au bon sens du terme. Ensuite, il appartient au décideur d'évaluer en toute intégrité de ce qu'il faut faire en confrontant tous les avis et en essayant de satisfaire le plus de gens possible. Ainsi, se déroule le processus démocratique.

Au cours de cette table ronde, nous aborderons plusieurs sujets. Le premier est celui-ci : le critère de rareté est-il pertinent? La notion de «maladies rares », telle un paquet commun et un ensemble de politiques, a-telle un sens ? Un cancer rare est-il plus proche des autres cancers ou d'autres maladies rares?

\section{Brigitte Chabrol}

Je parlerai plutôt d'une maladie neurologique rare par rapport à une maladie neurologique plus fréquente, ou d'un handicap très rare par rapport à un handicap d'origine plus fréquente.

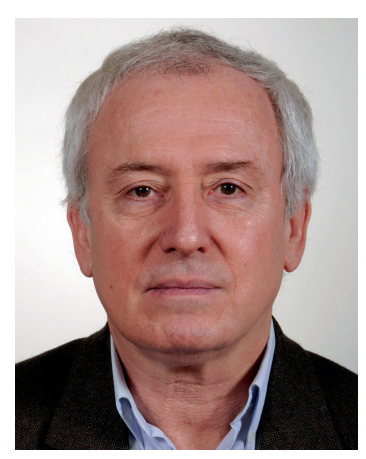

La table ronde est animée par Gilles Roche (Eurobiomed)

II faut surtout savoir le décliner dans la relation individuelle qui caractérise la relation médicale entre le patient et son médecin. Qu'il s'agisse d'une cause extrêmement rare ou plus fréquente, la démarche médicale doit rester dans des règles de base identiques pour tous, c'est-à-dire partir d'une réflexion pour tirer une hypothèse qui doit être confirmée avant d'établir un diagnostic, celui-ci constituant la plate-forme indispensable pour mettre en place une prise en charge. L'expérience et la compétence du médecin qui reçoit le patient sont très importantes pour définir une organisation des soins. La question doit être posée à ce niveau plus qu'à la rareté de la maladie.

Une maladie très rare est mal connue, alors qu'une maladie fréquente est plus connue. C'est vrai dans la population générale médicale. Comme je ne vois que des enfants porteurs de maladies très rares, mon domaine de compétence concerne beaucoup plus les maladies très rares que les maladies très fréquentes.

Par conséquent, un circuit bien déterminé et une très bonne orientation importent plus que la notion de rareté. Le médecin doit savoir reconnaître ses limites de compétence pour orienter le patient vers un circuit déterminé et lisible. 


\section{Gilles Roche}

Le critère «maladies rares » n'est donc pas fondamental, mais il ne faut pas le jeter non plus.

\section{Vololona Rabeharisoa}

Mon point de vue sera peut-être différent et complémentaire. Le critère de rareté est-il pertinent pour définir une politique publique spécifique à ces maladies ? Ma réponse sera en deux temps. En tant que critère d'action politique, la rareté est un critère pertinent. En effet, il a permis à des populations invisibles de devenir visibles, car on les a comptées. Or, la politique est une affaire de compte, au sens noble du terme : on compte les gens pour faire en sorte qu'ils comptent dans les affaires publiques. Le critère de rareté, qui est un critère épidémiologique conventionnel, permet de faire compter des problèmes qui longtemps n'ont pas été pris en compte.

Une fois que ce critère est établi comme critère de politique publique, la question de ce que cela produit se pose. Après plusieurs décennies de politiques spécifiques aux maladies rares, en particulier en France, il importe de s'interroger sur ce que ces politiques redistribuent vers la société, sous forme de bénéfices en matière de recherche, de gestion des affaires publiques pour d'autres catégories de pathologies et d'autres populations de malades.

Le critère de rareté est tout à fait pertinent, pour autant qu'il soit discutable.

\section{Anne-Sophie Lapointe}

Le critère de rareté est pertinent dans le sens où il répond à des besoins. Les besoins non couverts dans le champ des maladies rares sont plus importants que pour des maladies plus communes: errance de diagnostic, maladies peu connues, malades isolés, absence de traitements. Au cours de ces 15 dernières années, la prise en compte de ce critère de rareté et de ses conséquences a permis de répondre à des besoins. Les deux PNMR et le règlement de 1999 sur les médicaments orphelins ont pris en compte ce critère pour répondre aux besoins, notamment celui d'avoir des thérapies.

\section{Gilles Roche}

La notion de maladies rares a permis de les rendre visibles et de s'en occuper. Après avoir été négligés, les patients atteints de maladies rares seraient-ils paradoxalement devenus les enfants gâtés de la médecine, comme j'ai pu l'entendre dire de façon surprenante?

\section{Brigitte Chabrol}

L'expression « enfant gâté » est provocatrice et non adaptée car ces personnes sont atteintes d'une maladie rare. L'organisation des soins très particulière mise en place grâce aux deux PNMR assure une reconnaissance et une lisibilité bien meilleures. En outre, la distribution sur le territoire national a considérablement évolué ces dernières années. Par ailleurs, les organisations mises en place ont permis de renforcer des équipes. II ne faudrait pas que cette évolution s'opère au détriment d'autres équipes, car les ressources en termes de soins courants, d'équipes de soignants et de médecins sont limitées. Ce sujet est en lien avec la vision sociétale pour l'équité des soins entre malades.

\section{Anne-Sophie Lapointe}

Je m'insurge totalement contre l'expression « enfant gâté ». Les personnes atteintes de maladies rares ont une double peine. Ils sont non seulement malades, mais aussi la rareté de leurs maladies complexifie le diagnostic et les isole davantage.

Les soins liés aux maladies rares sont chronophages. La tarification à l'acte (T2A) n'est sans doute pas valorisée suffisamment pour ces actes spécifiques.

\section{Vololona Rabeharisoa}

L'expression « enfant gâté » pose la question de priorisation des problèmes de santé de façon provocatrice sans la traiter au fond. Les personnes qui fument, qui ont un cancer des poumons et qui sont traités pour cette maladie pourraient aussi être considérées comme des enfants gâtés de la médecine. Lorsque l'épidémie de Sida a commencé, d'aucuns considéraient que les malades n'auraient pas dû se livrer à certaines pratiques dont les conséquences médicales coûtent cher à la société.

Les personnes atteintes de maladies rares ne sont pas des enfants gâtés car elles ont été longtemps ignorées. Des efforts spécifiques sont désormais faits à leur endroit. La question de l'équilibrage des priorités publiques et de l'allocation des ressources s'en est trouvée transformée.

En effet, il faut reconnaître que ce qui a été fait pour les maladies rares, par exemple en termes de soins, a des effets sur la prise en charge d'autres pathologies. Les recherches que j'ai effectuées avec mes collègues montrent, par exemple, que des associations concernées par des maladies non rares se réfèrent à ce qui est fait pour les maladies rares, et réfléchissent à la transposition de certaines solutions, par exemple les centres de référence.

\section{Pierre Le Coz}

Pour prolonger ce qui a été dit, il faut rappeler que l'une des caractéristiques de la maladie rare est d'être souvent précédée par une période d'errance diagnostique qui retarde la prise en charge. Cet aspect a été fortement souligné par Marie-Hélène Boucand qui a soutenu cette année une thèse sur les maladies rares. L'enquête qu'elle a menée pendant trois ans a montré que les patients subissent des dommages psychiques spécifiques car leur maladie suscite autour d'eux peur, intrigue et incrédulité. Ils sont soulagés de savoir que leur maladie a un nom après une longue errance diagnostique. Après cette phase où ils retrouvent un peu espoir, les patients se rendent compte que les 
thérapeutiques sont généralement limitées. Ils épuisent leur temps et leurs ressources à essayer de se faire reconnaître comme des personnes à part entière au sein de la cité.

\section{Florence Bordon-Pallier}

La notion de rareté n'est pas pertinente d'un point de vue individuel, car un malade atteint d'une maladie rare ou fréquente est un malade. Du point de vue du traitement, le fait d'être atteint d'une maladie rare ou fréquente n'assure pas qu'elle puisse être traitée et que le traitement soit accessible.

\section{Gilles Roche}

Il existe des maladies rares et des maladies ultra-rares. Si la société a 100 euros à dépenser en recherche, en développement de produits ou en paiement des médicaments et la prise en charge, faut-il privilégier l'égalité de l'accès à la prise en charge individuelle de tous les patients, y compris ceux qui ont une maladie rare? Ou la société doitelle raisonner au niveau global en allouant ces 100 euros de manière aussi efficace que possible, probablement en mettant cette somme plutôt sur une maladie fréquente?

\section{Pierre Le Coz}

La valeur de justice admet deux composantes qui coexistent parfois avec difficulté : l'égalité et l'équité. L'égalité signifie que quels que soient son âge, sa maladie, son statut socio-économique, l'être humain a une valeur absolue. Notre dignité n'est pas tributaire des contingences. L'égalité implique que la société fasse des efforts pour réduire les disparités entre les bien-portants et les malades. La justice consiste donc à lutter contre les discriminations, à affaiblir les écarts entre les patients et les autres membres de la société.

La justice présente aussi une composante d'équité. Par exemple, si je suis professeur à la faculté de médecine, l'égalité signifie pour moi que tous les étudiants ont droit aux mêmes enseignements. Mais le sens de l'équité implique qu'à l'issue du concours, seuls les $10 \%$ qui auront le plus souffert et lutté contre la tentation du divertissement auront réussi. Grâce à l'étalon du mérite, il est possible de hiérarchiser les étudiants sans attenter au principe de justice.

Or, en matière de santé, il n'est pas possible d'utiliser le critère du mérite. Les égalitaristes-déontologistes mettent plutôt l'accent sur le fait qu'on ne peut jamais complètement calculer les conséquences, que celles-ci sont souvent aléatoires, qu'un effet microscopique produit par une recherche sur une maladie rare peut avoir un effet en cascade sur des maladies plus fréquentes.

De leur côté, les utilitaristes cherchent à produire le plus grand bonheur pour le plus grand nombre de personnes, ce qui conduit, dans le domaine de la santé, à réduire le plus possible de souffrances chez le plus grand nombre de patients. Le bonheur de tous n'étant pas possible, des décisions difficiles de priorisation doivent parfois être prises, en privilégiant par exemple les personnes qui ont une plus grande espérance de vie.

Le bon positionnement éthique se situe entre l'égalité que représente l'école de pensée déontologiste, et l'équité qui est représentée par celle des utilitaristes. La vision populationnelle de l'homme doit sans cesse être mise en balance par la vision personnalisée de l'homme pour éviter d'entrer dans une logique sacrificielle

\section{Gilles Roche}

L'approche utilitariste nous ramène rapidement à des raisonnements économiques. Or, les études d'efficience doivent inclure des questions qui ne sont pas purement économiques.

\section{Florence Bordon-Pallier}

Nous parlons de besoins médicaux, de traitements, des besoins des patients. Si j'étais dans un fauteuil roulant, je trouverais sans doute que les innovations permettant d'accéder à mon domicile, de pouvoir écouter de la musique et d'utiliser un GPS devraient être prises en compte dans l'évaluation médico-économique de ma maladie.

\section{Anne-Sophie Lapointe}

Je m'inscris beaucoup plus dans l'idée d'efficience que dans la logique du plus grand nombre. Pour être efficient, il faut travailler par rapport à la singularité de la personne et aux besoins pertinents par rapport à la maladie. Ces besoins et critères pertinents doivent être remontés par les personnes malades et leurs proches. Ces critères donneront la valeur et l'efficience du médicament. Ils doivent pouvoir être utilisés dans l'évaluation thérapeutique du médicament et ensuite lors de cette évaluation en vie réelle. Cette somme des singularités doit être appréhendée en prenant en compte cette somme d'expérience des patients. Le grand nombre n'apporte pas forcément la qualité du produit. Ce qui compte, c'est d'être assuré d'avoir de bons critères d'évaluation en dehors du critère principal d'innocuité du médicament (safety). Dans ce contexte, les données de qualité de vie des personnes malades devraient pouvoir être mieux prises en compte dans les évaluations d'efficacité des traitements thérapeutiques.

\section{Gilles Roche}

Nous fonctionnons avec une enveloppe de ressources finie. De nombreuses ressources ont été mobilisées pour les maladies rares. À l'échelle de la société, cette allocation de ressources a-t-elle été efficace? Les contribuables s'y retrouvent-ils?

\section{Vololona Rabeharisoa}

Des évaluations seront sans doute nécessaires pour répondre à votre question. Mais pour reprendre ce que Anne-Sophie Lapointe vient de dire, je trouve la notion 
de rareté intéressante car elle oblige à s'interroger sur la singularité de la situation de la personne, à la fois au plan médical et au plan social. Le vrai défi des systèmes de santé, mais aussi des systèmes d'éducation, est de faire en sorte que la singularité soit au cœur de l'action tout en se souciant de la transposition possible d'une action ou d'une solution vers d'autres situations. L'enjeu est de faire en sorte que la singularité d'une situation puisse nourrir des réflexions sur les solutions à mettre en place dans d'autres situations. Cette logique, qui peut paraître abstraite, est en train de se développer dans différents secteurs de l'action publique.

\section{Pierre Le Coz}

La singularité se reflète sur le visage. L'éthique commence lorsque nous levons les yeux sur le visage de l'autre : tel est l'enseignement qui nous a été légué par Emmanuel Lévinas. Dans l'univers de la santé, nous avons parfois tendance à raisonner en termes de retour sur investissement et à nous focaliser sur la traçabilité. La démarche médicale risque alors d'être dévoyée par l'intelligence administrative, parasitée par une logique gestionnaire et protocolaire. Le risque est alors de dissoudre l'humanité du patient dans des procédures et des items. Cette intelligence désincarnée nous conduirait presque à oublier que la personne a un visage. Quand une famille ne paie pas son électricité, un agent administratif n'aura pas tellement de difficulté à décider de faire couper le courant ; il en sera tout autrement pour le technicien qui se rendra sur place et croisera le visage de la mère et de ses enfants. Le médecin se retrouve parfois dans la même situation que le technicien. La responsabilité pour autrui est le cœur de métier du médecin et lui assigne sa vocation humaniste.

\section{Brigitte Chabrol}

Je souhaiterais revenir sur la question initiale relative à l'impact médicoéconomique. L'organisation des soins est prédéterminée. En revanche, l'évaluation de l'apport ajouté pour un malade et sa famille des prescriptions proposées. Un joli travail a été réalisé sur une population d'enfants polyhandicapés suivis à Rabat au Maroc sans kinésithérapie et sur une population d'enfants suivis à Paris avec des protocoles extrêmement clairs. En termes de qualité de vie, de rétraction de douleurs, les résultats observés chez les deux populations étaient comparables, voire plus positifs à Rabat. Au sein de familles marocaines totalement impliquées, les grands-mères continuaient à faire des massages avec de l'huile d'argan matin et soir. L'apport des sciences humaines doit être pris en compte dans les soins chroniques que nous mettons en place. Nous devons vraiment sortir d'un schéma classique.

\section{Gilles Roche}

Des ressources ont été allouées aux maladies rares. Pour autant, celles-ci ne sont pas toutes logées à la même enseigne. Si un enfant de star est atteint d'une maladie donnée, celle-ci bénéficiera d'une reconnaissance extraordinaire, voire de ressources financières supplémentaires. Les associations de patients qui n'ont pas de star ou qui n'ont pas su médiatiser leur cause disposeront de ressources plus limitées. N'y a-t-il pas là une certaine injustice?

\section{Anne-Sophie Lapointe}

La question de l'autonomie des associations se pose. II leur appartient d'être inventives pour aller chercher de nouveaux modes de financement. De jeunes associations inscrivent leur action dans une dynamique extrêmement positive, notamment à travers du crowdfunding. Les associations les plus connues sont présentes depuis la fin des années 1950.

\section{Gilles Roche}

Je pensais à la maladie de Charcot, avec le célèbre joueur de baseball Lou Gehrig. Sans cette célébrité, la collecte de fonds aurait été autrement plus difficile. Est-ce juste?

\section{Pierre Le Coz}

Le lobbying suscite certaines inquiétudes chez nos concitoyens qui ont l'impression que des décisions touchant l'intérêt général se jouent dans des couloirs, ou que les dossiers sont livrés à des rapports de force, à des relations intertribales. Le lobbying comporte la menace d'empêcher l'expression de la volonté générale, qui est le fondement de la loi dans un État de droit. Le groupe d'intérêt qui prédomine est celui qui parle le plus fort sur la place publique. Dès lors, l'universalité républicaine se trouve biaisée. Pour éviter ce travers, une éthique de la discussion - c'est-à-dire une bonne représentativité de tous les lobbyings - est nécessaire. II faudrait, de plus, veiller à la traçabilité des fonds et à l'équité de répartition des milieux associatifs pour pondérer le risque de disparités.

\section{Gilles Roche}

Le principe est intéressant, mais sa gestion pratique pose question.

\section{Anne-Sophie Lapointe}

En matière d'équité, nous avons besoin de locomotives. Certaines associations ont entraîné une dynamique positive dans le mouvement des maladies rares. Celui-ci se serait structuré de manière bien différente sans l'AFM-Téléthon à la fin des années 1980. L'AFMTéléthon a en effet beaucoup poussé les associations à se créer pour que les gens s'autonomisent et se prennent en charge par rapport à leur pathologie. Ce mouvement d'impulsion était très positif et le reste encore. Il est à noter que le règlement de 1999 pour le médicament orphelin doit beaucoup à l'action importante d'Eurordis. Eurordis a été créé en 1997 grâce à l'action conjointe de Vaincre la Mucoviscidose, la Ligue contre le Cancer, I'AFM-Téléthon et Aides. II y a un réel accompagnement et une transmission de savoirs et de 
connaissances entre les plus grosses associations et des plus petites. Les réunions inter-membres de l'Alliance Maladies Rares (Rime) servent à partager les informations et à former toutes les associations maladies rares qui le souhaitent.

\section{Gilles Roche}

Nous parlons de la responsabilité de la société vis-à-vis des patients qui ont une maladie rare. À l'inverse, les associations de patients ontelles une responsabilité vis-à-vis de la société ? Le militantisme d'un groupe peut-il aller contre l'intérêt global de la société ?

\section{Vololona Rabeharisoa}

Le lobbying est une activité professionnelle reconnue qui répond à une codification précise, sauf en France. Les lobbies sont des acteurs parmi d'autres, et il ne faut pas confondre lobbying et construction de nos intérêts collectifs. Ceux-ci se construisent ensemble par des discussions, des négociations et, parfois, des renoncements; ils ne sont pas posés tels quels devant nous. Aussi paradoxal que cela puisse paraître, je dirais qu'il en va de même de nos intérêts particuliers : ils ne sont pas donnés; nous les découvrons en discutant avec d'autres. Telle suggestion faite par un tel peut nous conduire à prendre conscience de nos intérêts individuels. Pour sortir des oppositions, il faut remettre au cœur de la discussion les modalités que nous nous donnons pour construire ensemble nos intérêts collectifs.

Les associations de patients ont bien sûr un devoir vis-à-vis de la société puisqu'elles sont les porte-parole des malades qu'elles réunissent. Les recherches que j'ai menées avec mes collègues montrent que les associations remplissent pleinement leur devoir vis-à-vis de la société : elles prennent leur part dans l'effort collectif, discutent au sein de comités des modalités de prise en charge des malades, réfléchissent à la portée de leurs actions.

Mais j'aimerais ajouter que chacun doit exercer ses responsabilités, en veillant à éviter que les uns se substituent aux autres. En particulier, les autorités publiques, dont je déplore l'absence à cette table ronde, ont un rôle à jouer et devraient contribuer à cette réflexion collective ; les cliniciens ont aussi leur rôle à jouer. La difficulté est de trouver les bonnes modalités pour définir ensemble qui fait quoi.

\section{Gilles Roche}

Nous avons invité plusieurs députés à cette table ronde, mais aucun n'a répondu présent. Les représentants de l'autorité publique ont été particulièrement absents à cette édition de RARE, alors qu'ils ont davantage participé aux éditions précédentes de RARE. Les autorités publiques sont dans une sorte de psychose à l'idée de se « compromettre » dans des événements auxquels participent des représentants de l'industrie pharmaceutique. Espérons que cet état d'esprit ne durera pas.

\section{Pierre Le Coz}

Au XIXe siècle, des sociologues et des philosophes se posaient la question de savoir si la démocratie naissante, basée sur la souveraineté de l'individu, n'allait pas s'atomiser et se démembrer. Tocqueville entre autres, s'est interrogé sur la construction d'une société qui ne repose plus sur les liens traditionnels de solidarité. Avec le recul, il semble que le foisonnement des associations a permis d'empêcher l'atomisation sociale. L'association a été le chaînon manquant entre l'individu et l'État. Certes, l'individu tend à délaisser l'intérêt commun, mais il peut encore s'intéresser à la société via des micro-groupes dans lesquels il s'implique.

Le milieu associatif peut toutefois conduire à des excès lorsqu'il est traversé par des courants militantistes. Ainsi, lors des débats sur la loi de bioéthique en 2004, certaines associations ont cherché à inciter le législateur à autoriser le clonage thérapeutique, qui était censé affranchir les patients de toutes sortes de maladies incurables. Aujourd'hui, plus personne n'évoque cette solution. Par ailleurs, le déficit de recherche sur les embryons a été, à l'époque, présentée comme un obstacle à la mise au point de nouvelles thérapeutiques. Or, on sait que, depuis la fin des années 1990, l'Angleterre n'a pas fait de progrès significatifs dans la mise au point de thérapeutiques à partir de recherche sur l'embryon. Il apparaît ainsi, avec le recul, que les difficultés que rencontrent les chercheurs ne tiennent pas au simple fait que la loi pose des limites. Il ne suffit pas de changer la loi pour changer le destin des malades.

\section{Gilles Roche}

La société française alloue un certain nombre de ressources aux maladies rares pour la recherche fondamentale, le développement de médicaments et de moyens de prise en charge, et la prise en charge des produits à disposition. Les ressources allouées ont-elles permis de répondre aux vrais besoins médicaux?

\section{Florence Bordon-Pallier}

La recherche et le développement avancent avec des hypothèses que les chercheurs testent. Les ressources ont bien été utilisées puisque les outils mis en place permettent d'augmenter le niveau de connaissance. En outre, les chercheurs ont à leur disposition de nouvelles hypothèses à tester qui pourraient mener à de nouvelles thérapeutiques permettant d'améliorer la qualité de vie des patients. J'ai l'impression que nous sommes aujourd'hui dans la bonne dynamique.

\section{Brigitte Chabrol}

Au niveau de l'organisation hospitalière, la tarification particulière qui est appliquée incite les équipes médicales à avoir des attitudes quelque peu «schizophréniques ». Au cours d'une même consultation de 
30 minutes, elles peuvent en effet passer d'un paiement à l'acte puis à une MIG ; et bientôt elles appliqueront le schéma pour les handicaps rares. La situation est donc complexe. L'argent dépensé a permis d'améliorer l'état des hôpitaux publics et l'organisation des soins, ce qui profite à l'ensemble des patients. L'évaluation finale de toutes ces actions - en vue de leur reconduite - me semble particulièrement complexe.

\section{Vololona Rabeharisoa}

La question de la bonne allocation des ressources est une question classique d'économie politique ; elle n'est pas propre aux maladies rares. Cette question renvoie à une seconde question : la population générale a-t-elle un égal accès aux services de soin ? Ma réponse à cette question sera nuancée. En France, l'accès aux soins est probablement sans égal par rapport à d'autres pays. Cependant, tout dépend de ce qu'on appelle soin, et ici, je voudrais prolonger les remarques de Brigitte Chabrol.

L'un de mes anciens doctorants, Pierre-André Juven, a réalisé une thèse remarquable sur l'histoire de la T2A dans les hôpitaux publics et privés en France, thèse pour laquelle il vient d'obtenir le Prix Le Monde de la recherche universitaire 2015 en sciences humaines et sociales et qui sera publiée aux Presses Universitaires de France. II a en particulier étudié une action menée par l'association Vaincre la Mucoviscidose qui s'était émue du fait que la T2A avait tendance à jouer en défaveur d'activités de soins non techniques et non réalisées à l'hôpital en présence du malade, par exemple la coordination de l'équipe infirmière avec l'école, l'éducation thérapeutique du patient, l'accompagnement des familles. Ces soins (au sens de «care » en anglais) ne figurent dans aucune comptabilité. Les ressources sont probablement bien allouées si l'on s'en tient aux actes techniques. Mais si on considère l'ensemble des activités nécessaires à une bonne prise en charge des patients, dont les effets sur la qualité de vie et le bien-être au quotidien sont importants, on peut légitimement se demander ce à quoi les ressources ont été employées.

\section{Anne-Sophie Lapointe}

Compte tenu des contraintes budgétaires actuelles, les ressources consacrées au développement de médicaments doivent être allouées sur la base de critères pertinents pour le malade; ainsi, le gâchis dans des essais cliniques mal construits serait évité et l'efficacité du médicament pourrait mieux être évaluée.

\section{Pierre Le Coz}

En dehors des questions d'allocations des ressources, on doit tenir compte des bénéfices symboliques. Par exemple, en novembre 2005, une greffe de la face a été réalisée dans le Nord de la France. Si on raisonne en termes utilitaristes, on dira que les millions d'euros investis dans cette opération auraient pu être utilisés pour résorber une plus grande quantité de souffrances. Au lieu de faire ce calcul, l'équipe médicale a pris en compte la singularité de la personne; elle l'a envisagée sous l'angle de sa dignité, de sa valeur inconditionnelle. À travers cette opération, la société s'est adressée à elle-même un message : elle est capable de faire preuve de démesure par amour pour l'humanité.

\section{Gilles Roche}

Quand on fait venir un enfant d'Afrique subsaharienne pour l'opérer d'une cardiopathie congénitale, on peut se demander si la même somme pourrait être utilisée à nourrir des enfants et à traiter des diarrhées infectieuses. Le débat sur l'allocation des ressources est compliqué car l'arbitrage entre l'individu et le groupe est difficile.

\section{De la salle}

On parle beaucoup des fonds consacrés à la recherche et aux médicaments, mais qu'en est-il des aides pour le quotidien des patients et de leur famille?

\section{Gilles Roche}

Au cours de notre discussion, nous n'avons pas fait de distinction entre la recherche, le développement, le remboursement des médicaments, le diagnostic, etc. Nous n'avons pas exclu les aides pour le quotidien.

\section{Pierre Le Coz}

Effectivement, l'amélioration de la santé de la population ne se réduit pas à la recherche biomédicale et à la distribution de médicaments; elle doit conduire à mettre en œuvre tous les moyens susceptibles d'apaiser les maux du quotidien (ergothérapie, aménagements urbains, etc.). Selon la définition de la santé donnée par l'Organisation mondiale de la santé, la santé est « un état complet de bien-être physique, mental et social ». Il s'agit d'une définition maximaliste, peut-être un peu trop ambitieuse. La définition de la santé par la négative comme « absence de maladie ou de handicap » pourrait déjà constituer un louable objectif. Si les hommes pouvaient déjà s'en tenir à un principe de non-malfaisance, c'est-à-dire se dispenser d'aggraver la souffrance que la vie se charge de leur imposer, ce serait déjà un grand progrès pour l’humanité.

\section{De la salle}

Le mot « démocratie » n'a pas été prononcé au cours de cette table ronde.

\section{Vololona Rabeharisoa}

En philosophie politique, la démocratie revêt deux sens. Le premier est l'égal accès de tous aux biens communs. Le second pose la question de la prise en compte de la voix de tous dans la fabrication des biens communs. Le critère de rareté est très intéressant parce qu'il lie ces 
deux définitions. Il a permis à une population longtemps à la marge des espaces communs d'accéder à la connaissance et à la reconnaissance, à la médecine, à la citoyenneté pleine et entière. Dans le même mouvement, le critère de rareté a constitué un ferment très important de la démocratie au second sens que je viens de donner, c'est-à-dire qu'il a permis de donner la parole et de faire compter des individus qui étaient jusqu'ici sans voix. Nous devrons, je crois, faire advenir davantage encore cette seconde définition de la démocratie afin d'entendre ce que les personnes à la marge de nos espaces communs ont à nous dire. Il s'agit là du plus beau défi que nous offrent les personnes atteintes de maladies rares.

\section{Brigitte Chabrol}

Cette table ronde reflète très bien l'intérêt du «travail ensemble ». Chacun dans son domaine de compétence permet à tous d'avancer dans le même sens. Le travail mené avec les associations depuis ces dernières années a permis aux médecins de les connaître. Les associations ne sont pas uniquement des acteurs de lobbying. La connaissance du quotidien des patients permet d'essayer de les soulager au mieux.

\section{Florence Bordon-Pallier}

Il est très important de souligner que nous travaillons tous ensemble. Les entreprises ont appris à travailler avec des associations de patients. Les premières n'apportent pas que des traitements aux maladies, mais elles offrent aussi une écoute et une aide à tous les acteurs qui accompagnent les patients.

En tant que partie intégrante de la société, les entreprises ont une responsabilité. Notre entreprise base sa responsabilité sociétale sur plusieurs piliers, et notamment la planète, l'éthique (en particulier dans les essais cliniques) et les patients.

\section{Gilles Roche}

Il est bien de rappeler que les entreprises prennent très au sérieux leur responsabilité sociétale et dédient des équipes à ce domaine.

Nous arrivons au terme de cette table ronde, et nous remercions chaudement nos experts pour leur engagement, et la salle pour sa participation. $\diamond$

\section{LIENS D'INTÉRÊT}

Florence Bordon-Pallier déclare être salariée de l'entreprise SanofiGenzyme.

G. Roche, V. Rabeharisoa, B. Chabrol, P. Le Coz, A.S. Lapointe déclarent n'avoir aucun lien d'intérêt concernant les données publiées dans cet article. 